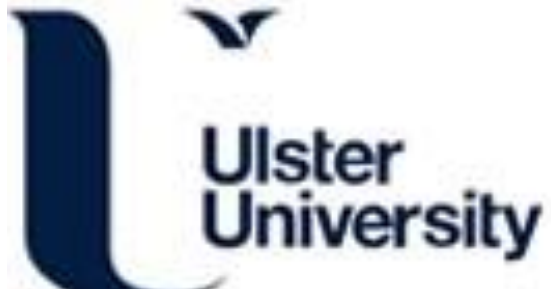

Application of synthetic patient data in the assessment of rapid rule-out protocols
usingPoint-of-Care testing during chest pain diagnosis in a UK emergency department

Robinson, S., FitzGibbon, F., Eatock, J., Hunniford, T., Dixon, D., \& Meenan, BJ. (2009). Application of synthetic patient data in the assessment of rapid rule-out protocols usingPoint-of-Care testing during chest pain diagnosis in a UK emergency department. Journal of Simulation, 3, 163-170. https://doi.org/10.1057/jos.2009.4

Link to publication record in Ulster University Research Portal

Published in:

Journal of Simulation

Publication Status:

Published online: 07/09/2009

DOI:

10.1057/jos.2009.4

Document Version

Publisher's PDF, also known as Version of record

\section{General rights}

Copyright for the publications made accessible via Ulster University's Research Portal is retained by the author(s) and / or other copyright owners and it is a condition of accessing these publications that users recognise and abide by the legal requirements associated with these rights.

\section{Take down policy}

The Research Portal is Ulster University's institutional repository that provides access to Ulster's research outputs. Every effort has been made to ensure that content in the Research Portal does not infringe any person's rights, or applicable UK laws. If you discover content in the Research Portal that you believe breaches copyright or violates any law, please contact pure-support@ulster.ac.uk. 


\title{
Application of synthetic patient data in the assessment of rapid rule-out protocols using Point-of-Care testing during chest pain diagnosis in a UK emergency department
}

\author{
S Robinson*, F FitzGibbon, J Eatock, T Hunniford, D Dixon and BJ Meenan ${ }^{1}$ \\ University of Ulster, Northern Ireland, UK
}

Clinical research is often delayed by the lack of data and the need for ethical approval. We suggest that this need could be initially satisfied by synthetic data that has the same characteristics as those from patient records. The generation of this data requires some domain knowledge to ensure appropriate data management. As an exemplar of this concept we generate patients presenting with undifferentiated chest pain at Emergency Department (ED). Their diagnosis uses biochemical markers indicative of myocardial cell damage. Efficient diagnosis is paramount and a number of different competing protocols have been advocated. Analysis of resulting data shows that while the measurement of cardiac markers may not register above a cut-off value that the time differentiated rule-out protocols are valuable indicators of disease. We therefore demonstrate both concept and value of the use of synthetic data that would have taken years to gather and not have been reproducible or repeatable.

Journal of Simulation (2009) 3, 163-170. doi:10.1057/jos.2009.4

Keywords: synthetic data; point-of-care; rule-out; chest pain; emergency department

\section{Introduction}

Clinical data is a valuable commodity and difficult to obtain. Often the reason being espoused for the non-proliferation of data are the ethical issues associated with patient information. The incentive to share the object of their research should be good science. Good science should be reproducible and verifiable but how can this be if the original data is not made available to others who may wish to understand how and from where the summary statistics were derived. The NHS (www.connectingforhealth.nhs.uk/systemsandser vices/infogov) and the Ministry of Justice have deliberated on these issues and have recommended in the Data Sharing Review (www.justice.gov.uk/docs/data-sharing-review-report. pdf) that there is a need to develop mechanisms whereby research and statistical analysis for public benefit can be carried out while still safeguarding the privacy of individuals.

The aim of this paper is to explore a technique of using machine generated patient data for the investigation of clinical procedures. Our eventual aim is to be able to produce synthetic patient data sets with more variables,

\footnotetext{
*Correspondence: $S$ Robinson, Multidisciplinary Assessment of Technology Centre for Healthcare (MATCH), Nanotechnology \& Integrated Bioengineering Centre, Faculty of Engineering, School of Electrical and Mechanical Engineering, University of Ulster, Shore Road, Newtownabbey, County Antrim, Northern Ireland, BT37 0QB, UK. E-mail: dobbyr@googlemail.com
}

which have the same statistical properties and interdependencies as the original sample data set. The essential feature of these data is that they do not relate to any individual patient (unlike re-sampling methods) but are entirely artificial. As such, confidentiality and ethical issues will not apply.

We are not advocating that simulations fed with synthetic data should form the basis for policy changes in patient care. Rather, that those simulations can be used to explore procedures; the economic value of novel medical devices can be evaluated and that researchers can hone their hypotheses in a virtual world before the clinical trials are conducted live. Simulation is increasingly being used to study patient pathways (Lattimer et al, 2004) and to study diagnostic practice using a manikin with appropriately generated symptoms (Brendan et al, 2002; DeVita et al, 2005). On a systems scale, the East of England SHA in collaboration with Simul8 corporation have created a Scenario Generator - a simulation tool that will facilitate investigation into the logistics of patient access, staffing and costing (www.healthcareworkforce.nhs.uk/workforceplannermenus/ index.php?option $=$ com_docman\&task $=$ doc_view\&gid $=3$ ). Only recently has synthetic patient data been used to feed such simulations (Dutta et al, 2005; McCabe et al, 2008). We believe that this is one possible way to assist the design of experiments and clarify the process that will lead to obtaining ethical approval for clinical trials. The freedom from the ethics restrictions imposed will, at least in part, 
remove the disincentive to share data and thereby encourage knowledge generation through openness of research findings.

This study presents an exemplar case for patients presenting at ED with chest pain, a scenario that is of great clinical and economic importance to the NHS. An estimated 500000 patients present to ED with chest pain each year, which is $25 \%$ of all admissions and at a cost of some $£ 15$ billion (Fox, 2005; Rajappan et al, 2005). However, a general analysis has shown that some two-thirds of presenting patients have non-cardiac chest pain. Of those that are cardiac related, $40 \%$ of these patients will have low risk of Acute Myocardial Infarction (AMI) (Turab et al, 2006). Although it is clear that misdiagnosis incurs additional cost and jeopardises patient wellbeing, these patients may have to be admitted due to insufficient clinical evidence to allow their safe discharge. This contributes a significant and unnecessary cost burden to the NHS. Furthermore, Turab et al (2006) reported that between 2 and $10 \%$ of the presenting patient group are inappropriately discharged with undiagnosed AMI.

There is a need for an improved rapid rule-out diagnostic test to differentiate these patients and contribute to reducing hospital admission costs. Benger et al (2002) cites that in the United Kingdom 300000 people suffer an acute heart attack each year, of whom $50 \%$ die. Of those, about one-third of all deaths occur within the first hour (NICE, 2002) giving a narrow time window for diagnosis and intervention. Any time delay taken to initiate treatment is therefore significant. A preferred treatment of AMI is with thrombolytic drugs administered intravenously. This therapy operates by dissolving the offending clot formation in the coronary artery; however its effectiveness is greatest when it is administered early (Carley, 2002; Heath et al, 2003). However, there are risks associated with thrombolytic therapy and it must only be administered to those with confirmed AMI and only then with caution. Accurate and rapid diagnosis is therefore highly desirable. Efficient diagnosis of AMI is paramount and the most effective identifiers of myocardial cell damage are the presence of the cardiac markers myoglobin, troponin I and CK-MB in the blood. These proteins that originate within the cells of the heart can be measured by a Point-of-Care device (PoC) and if found to be elevated are strong evidence of cellular damage. The testing for the cardiac marker troponin has become a gold standard of AMI diagnosis rather than just another indicator.

The essential advantages of identifying the elevation of cardiac markers with $\mathrm{PoC}$ is the rapidity of the test turnaround time and the resultant seamless and, consistent approach to coronary care. The turnaround time is typically between 12 and $20 \mathrm{~min}$ for $\mathrm{PoC}$ as compared with about an hour for a pathology laboratory, depending on proximity and staff shift times. For cardiac patients this time difference could be vital. It will allow ED staff to accurately identify those patients that can start treatment immediately, thus increasing their chances of survival.

A further significance of this rapid turnaround time is that it provides for the possibility of alternative protocol pathways that otherwise would not be practical. A number of different rule-out protocols have been advocated for the diagnosis of patients using PoC (McCord et al, 2001; $\mathrm{Ng}$ et al, 2001) in which the time differential of cardiac marker levels is recorded and if it varies for any of the cardiac markers by more than $25 \%$, this can be deemed as evidence of ischemic processes. By rule-out is meant to establish beyond reasonable doubt that the patient is not AMI. The relative benefits of these rule-out protocols have been much debated (Goodacre et al, 2004, 2005; Collinson et al, 2006; Body, 2008). Consequently, the protocols present a paradigm shift from the early diagnosis of AMI. This study seeks to obtain data that can quantify the clinical benefit of PoC in cardiac screening. In this study we assess the value of these alternative $\mathrm{PoC}$ protocols using synthetic patient data generated with the same statistical characteristics as that of the sample data set.

The results of the simulation study shows that while the measurement of cardiac markers may not register above a cut-off value before $6 \mathrm{~h}$ after onset, that the rule-out protocols outlined by $\mathrm{Ng}$ et al (2001) and McCord et al (2001) could be valuable indicators of cardiac necrosis. The length of the time differential is important suggesting a diminishing return of sensitivity with delay. Experimentation shows that the sensitivity of the rule-out test is $94.9 \%$ at $90 \mathrm{~min}, 98.4 \%$ at $120 \mathrm{~min}$ and $99.0 \%$ at $180 \mathrm{~min}$.

Thus this paper demonstrates the value of synthetic data. Although it would have taken years to gather such data, it would not have been reproducible or repeatable. Such data would permit free exchange of information without the need for ethical approval. It is anticipated that the future will see synthetic data generation that handles more variables retaining interdependencies and passing the 'Turing test' (http://en.wikipedia.org/wiki/Turing_test) for clinical data.

\section{Materials and method}

The seed data for this study comprise the records of 137 patients that presented with chest pain at the ED at the Royal Victoria Hospital, Belfast between October 2007 and January 2008. The data recorded includes aspects of their medical history; arrival times; details of the patient diagnostic pathway and results of those tests; their preliminary diagnosis; ED departure time; hospital admission or otherwise and if they were admitted, the date of release from hospital and discharge diagnosis. Cardiac markers are tested using a Biosite Triage PoC device. These data are supplemented with summary statistics from a related study of 546 patients at the same hospital between 2004 and 2005 (Hamilton et al, 2008). These data can be 
assumed to have the same demographic characteristics as our data taken at a later date. Information is also sequestered from other literature (Goodacre et al, 2007).

The aim is to assess the likely benefits of different AMI rule-out protocols that have been suggested in the literature using simulated patients. The patient generator tool uses Weibull distributions for troponin levels in the blood for diseased and non-diseased patients. The parameters generated for each patient are a subset of those that would generally be taken at triage on entry to ED. The model assumes a likelihood of a patient being AMI as the prevalence in the population of $10.8 \%$ (Hamilton et al, 2008). Given that the patient is positive for AMI, the model generates a severity index from a parametric distribution of troponin values from our diseased population. If the patient is not diseased, the troponin level is similarly selected from the distribution for healthy patients.

The temporal profiles of these cardiac marker are taken from an internally published presentation (Adams, 2008). The cardiac markers are released from heart muscle cells as they die. There are a number of such profiles published showing the rise of cardiac markers and this representation has a good conformity with the authors published works. As can be seen from Figure 2 the time profile of each marker has a characteristic delay before it is detectable and therefore would not give a positive result with the $\mathrm{PoC}$ device until levels increase to the threshold for that device. For example, troponin levels are raised within 4-6h after onset of chest pain, peak at $12-20 \mathrm{~h}$ and remain elevated for 3-10 days. The elevation of the cardiac markers will depend on the severity of the disease and each one, as indicated in Figure 2, will remain proportionate to other markers for a given severity and time from onset. It can be seen from this profile that it is therefore important to know when the onset of AMI occurred. This value has been recorded for the patient data record and the model notes the delay after the time of onset that the blood sample is taken for the cardiac marker.

\section{A Judicious use of PoC}

As the speed of diagnosis of chest pain is critical to prevent further cardiac damage, the objective is to accurately and efficiently assess the likelihood of AMI. The cardiac markers however, may not be elevated and will not reach a cut-off value for at least $3 \mathrm{~h}$ after onset. Until that time it is not appropriate to test for cardiac markers. Traditionally the cut-off value for troponin at which a patient is considered to be diseased is arbitrarily set at the 99th percentile of the level of a healthy population (Apple et al, 2005). Although this definition is widely adopted, the critical value may vary with demographics (Apple et al, 2003).

A number of authors have experimented with variations of an AMI rule-out protocol (McCord et al, 2001; $\mathrm{Ng}$ et al, 2001; Dunn et al, 2006). They argue it has many advantages over the traditional blood test for cardiac markers that use a predefined critical cut-off value. The proposed rule-out protocols are less concerned with the time of onset or critical values. Using $\mathrm{PoC}$ to assess the three cardiac markers (myoglobin, troponin I and CK-MB) on arrival an initial base value is obtained. If it is within the normal range, then a further blood test is taken at a later time, for example $120 \mathrm{~min}$. If any of the cardiac markers are elevated from the base value by greater than $25 \%$, even if those values are within their normal range, then this is evidence that cardiac cell damage is in progress and the patient is admitted. The rule-out along with other diagnostic tests allows hospitals some confidence in discharging patients as cited by Dunn et al (2006) who reported some $40 \%$ reduction in admissions with this protocol.

\section{The model}

The aim of this exemplar model is to compare the effectiveness of a current chest pain protocol with what has become know as the 90 min rule-out $(\mathrm{Ng}$ et al, 2001) the $120 \mathrm{~min}$ rule-out (Dunn et al, 2006) and to explore a $180 \mathrm{~min}$ rule-out. The characteristics of the synthetic data are designed to conform to those of the data sample that has been collected from hospital records of the Royal Victoria Hospital (RVH). The data generation draws from empirical knowledge obtained from the live data set and reasoned principles of the parametric properties of those data guided by domain knowledge. The data is also augmented from similar studies and in particular summary data from previous work by Hamilton et al (2008). The data related to the same demographic population of patients at $\mathrm{RVH}$ entering ED with chest pain.

From the above research we may take the population prevalence of the cardiac disease (0.108), the sensitivity $(0.789)$ and the specificity $(0.967)$ for Troponin $\mathrm{T}$. Using Equation (1) and (2), this information permits the construction of the matrix in Table 1 showing the probabilities of a correct diagnosis of a patient's condition given a positive or negative test result for troponin.

$$
\begin{aligned}
\text { Sensitivity }= & \frac{\text { Number of True Positives }}{\text { Number of True Positives }} \\
& + \text { Number of False Negatives } \\
\text { Selectivity }= & \frac{\text { Number of True Negatives }}{\text { Number of True Negatives }} \\
& + \text { Number of False Positives }
\end{aligned}
$$

Using the PoC device for the detection of troponin, the cut-off of value is $0.09 \mathrm{ng} / \mathrm{ml}$ above which the patient is deemed to be AMI is which corresponds to the 99th percentile of the healthy population distribution (Apple et al, 2003). It is also know that the healthy population typically has a troponin profile described in Figure 1. The shape of the 
Table 1 Confusion matrix derived from Equations 1 and 2

\begin{tabular}{lccc}
\hline Test & Diseased pop. & Healthy pop. & Total \\
\hline$+\mathrm{ve}$ & 0.085 & 0.029 & 0.114 \\
-ve & 0.023 & 0.863 & 0.886 \\
Total & 0.108 & 0.892 & 1.00 \\
\hline
\end{tabular}

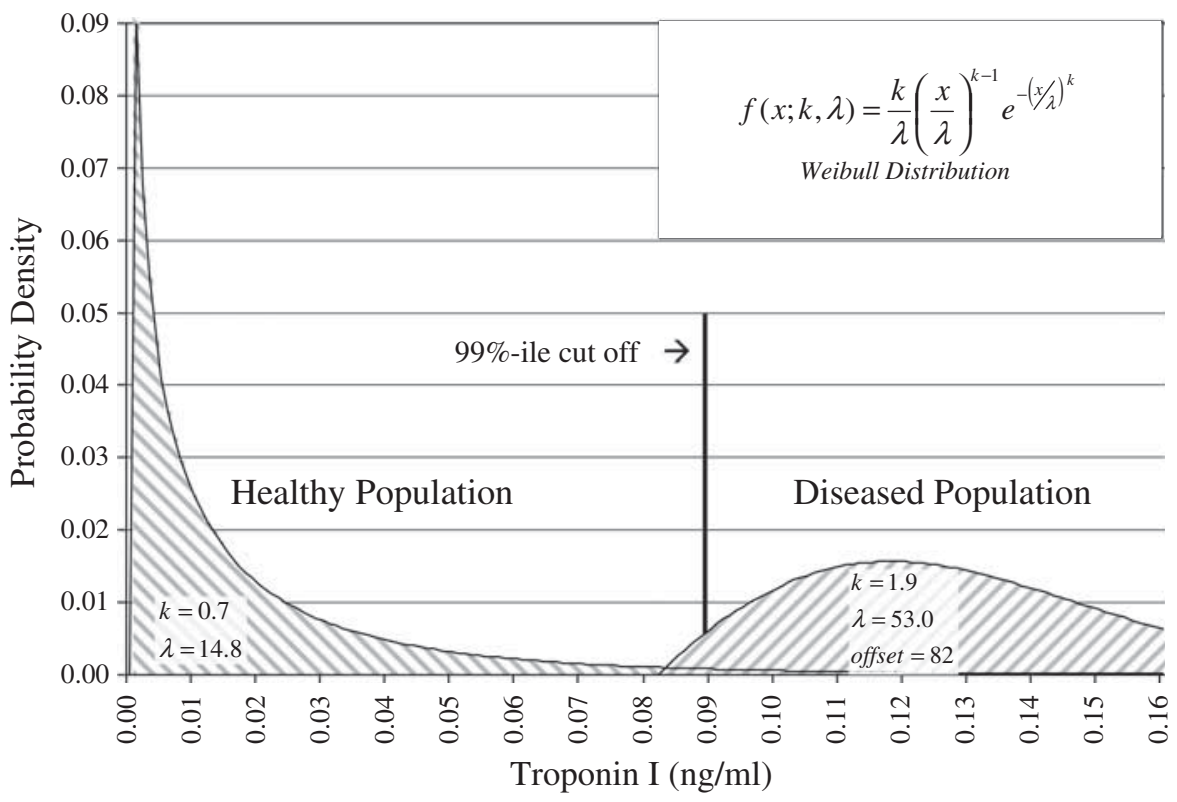

Figure 1 Probability distribution of troponin I in healthy and diseased population.

distribution to the left of the cut-off point is not of great importance here, but its similarity to a Weibull distribution is noted. This distribution is generally used in reliability analysis and time-to-failure profiles in a variety of contexts. As cardiac marker enzymes are an indicator of cardiac cell death (component failure), which must occur occasionally even in a healthy person and that blood troponin levels are a direct consequence of those cell deaths, then it would seem appropriate to use this distribution to describe the troponin occurrence in both the healthy and the diseased population.

Although the distribution of troponin levels from the available records of the diseased population bears a reasonable likeness to the Weibull distribution, it is not so easily parameterised with such low recorded values. This distribution has a correction factor added so as to be calibrated against the reported sensitivity found using the PoC device that was used at the optimum time for troponin detection. The ED staff intent was to take a blood test some $6 \mathrm{~h}$ after onset though the exact timing cannot be certain.

The simulation interprets this distribution as an index of the likely severity of AMI and is used to attenuate the amplitude of the cardiac marker values found from the cardiac marker profiles given in Figure 2. The matrix (Table 1) shows that the diseased population has a probability of 0.023 of being mistakenly identified as healthy (false positive). That is $2.3 \%$ of the diseased population will give values below that of the cut-off value. The data from the hospital records shows a diseased troponin distribution similar to that in Figure 1.

Each of the cardiac markers has a time related profile with hours $=0$ at the time of onset of the chest pain (Figure 2). The first marker to become evident is myoglobin after approximately $2 \mathrm{~h}$, likewise CK-MB after $4-6 \mathrm{~h}$ and troponin I after $4-8 \mathrm{~h}$, each reaching a peak before slowly declining. Figure 2 also shows the cut-off values for each of the cardiac markers.

Patient records show the time of onset of chest pain and the time of arrival. These values are plotted in Figure 3 and show some anomalies. From these data it appears that patients who experience symptom onset between 18:00 and 21:00 will, on average, wait more than twice as long before attending ED than those patients whose onset is between 22:00 and 13:00. This bimodal distribution of delays is described with two Poisson distributions also shown in Figure 3. To obtain a probability density function, the two distributions are summed and the result normalised. The rationale for this is that this distribution is often used to describe random queuing events. It would be tempting to surmise that those patients with the most acute symptoms of AMI would attend ED without delay. Although a severe 


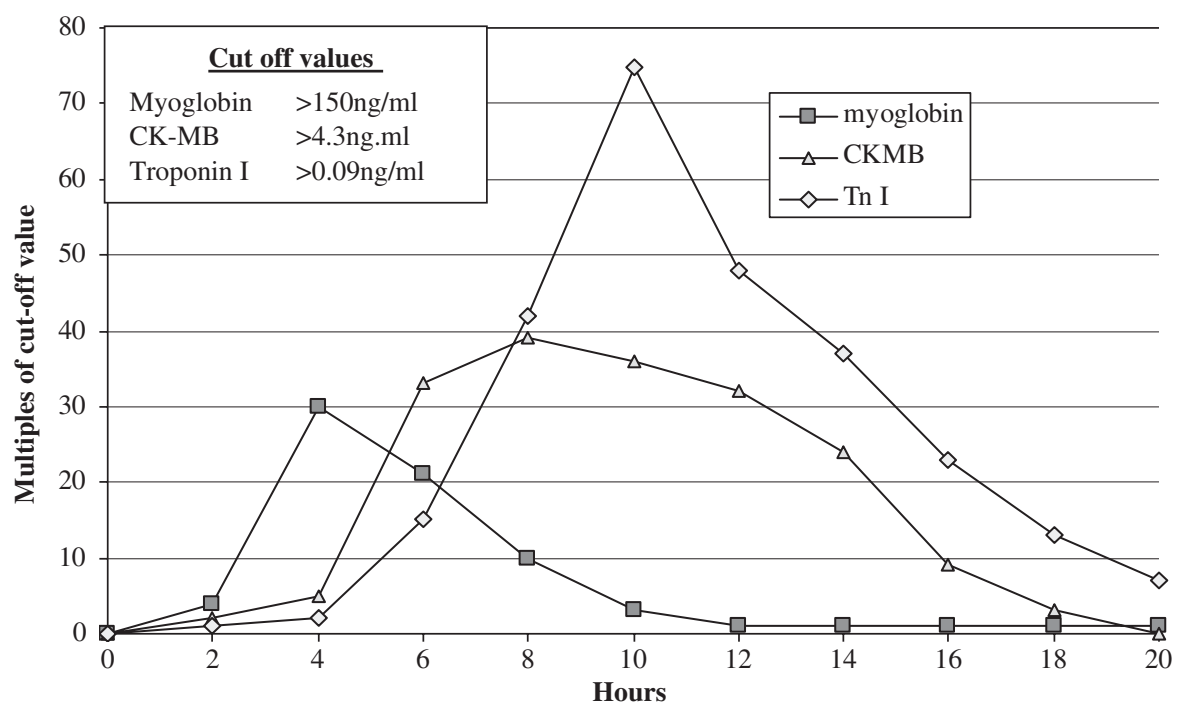

Figure 2 Temproal profile of cardiac markers. (P. Adams Newcastle Hospitals NHS Trust).

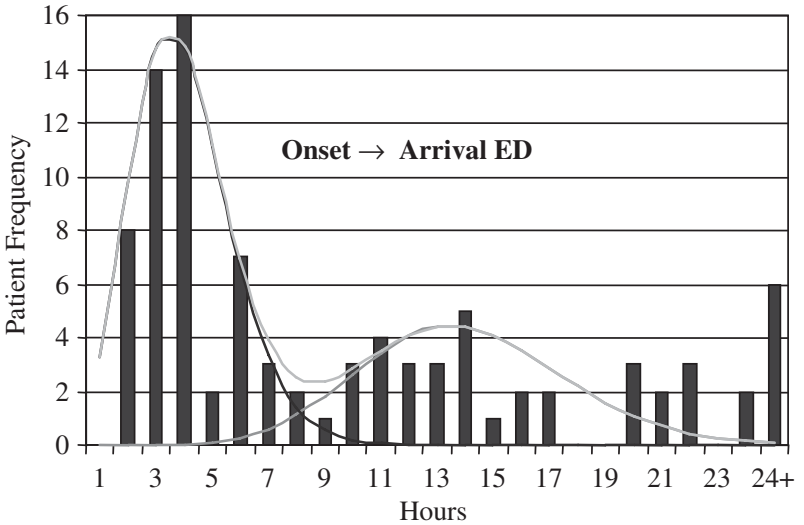

Figure 3 Distribution of delays between chest pain onset and presentation at ED.

attack should elicit an immediate transfer to hospital, there is no evidence for this in the data. One relatively severe case waited 3 days before attending ED. The profile of the diurnal cycle of ED attendance on the other hand is a welldefined bell curve with a mean of 15:00. It would appear therefore that people wait for a convenient time to attend ED. As a note of caution however, the delay between onset and arrival at ED is not measured by hospital staff; it is information volunteered by the patient. Although it is accepted that this time cannot therefore be verified, it is the only data that the hospital have to assess the progress of the AMI and is therefore used in this model.

The simulation involves the generation of a series of patient instances that have a time delay since chest pain onset taken as a random variable from the distribution described in Figure 3. This time value is used as an index to obtain the cardiac enzyme profile from Figure 2 for that time. A further random variable is used to select a severity for the AMI from the diseased population distribution in
Figure 1. This value is used to attenuate the values of the cardiac enzymes established above. With this method it is possible to create a realistic stochastic representation of the cardiac marker values for each patient with respect to the delay since onset. The diagnostic model receives this data as input. The simulation records the troponin level at the time of arrival and at 90, 120 and $180 \mathrm{~min}$ after arrival. If any of the readings are above the critical threshold (see Figure 2) for any of the markers or the subsequent reading shows more than a $25 \%$ increase in troponin I, CK-MB or myoglobin than the first reading, then the patient is regarded as having AMI.

For the AMI positive patients, one thousand patients were generated for each of the $90 \mathrm{~min}$ rule-out, the $120 \mathrm{~min}$ rule-out and the $180 \mathrm{~min}$ rule-out protocols. The possible outcomes for each patient are that they were diagnosed at the first blood test or at the second blood test or, if both were negative; then the results of the two tests are compared to see if any of the cardiac markers in the second test are more than $25 \%$ more or less than the first test. It is noted that because of the excessive delay of ED presentation after the chest pain onset, the cardiac markers may be on the decline. Regardless, the $25 \%$ change is recorded as an AMI indicator. The experiment is repeated 100 times.

\section{Results and discussion}

The model creates a series of patients with a $10.8 \%$ probability of AMI. For those deemed to have AMI, the delay since the onset is assigned to the patient from the distribution in Figure 3. That value indexes the distributions in Figure 2 to give the level of cardiac markers. The severity of disease is chosen from the disease profile in Figure 1. Those patients that are healthy are taken to have a troponin I 
Table 2 Showing the percentages diagnosed as AMI at the first test, second test or by $25 \%$ elevation of cardiac markers at second test. Also shown, those patients for whom AMI was not diagnosed

\begin{tabular}{|c|c|c|c|c|c|c|c|c|}
\hline \multirow[b]{2}{*}{ Minutes } & \multicolumn{2}{|c|}{ Test 1 (presentation) } & \multicolumn{2}{|c|}{ Test 2 (rule-out interval) } & \multicolumn{2}{|c|}{$25 \%$ elevation } & \multicolumn{2}{|c|}{ False negative } \\
\hline & Mean (\%) & $S d(\%)$ & Mean (\%) & $S d(\%)$ & Mean $(\%)$ & $S d(\%)$ & Mean (\%) & $S d(\%)$ \\
\hline 90 & 26.2 & 1.5 & 3.0 & 0.5 & 65.8 & 1.5 & 5.1 & 0.7 \\
\hline 120 & 26.4 & 1.3 & 5.6 & 0.7 & 66.5 & 1.4 & 1.6 & 0.4 \\
\hline 180 & 26.1 & 1.2 & 8.4 & 0.9 & 64.5 & 1.4 & 1.0 & 0.3 \\
\hline
\end{tabular}

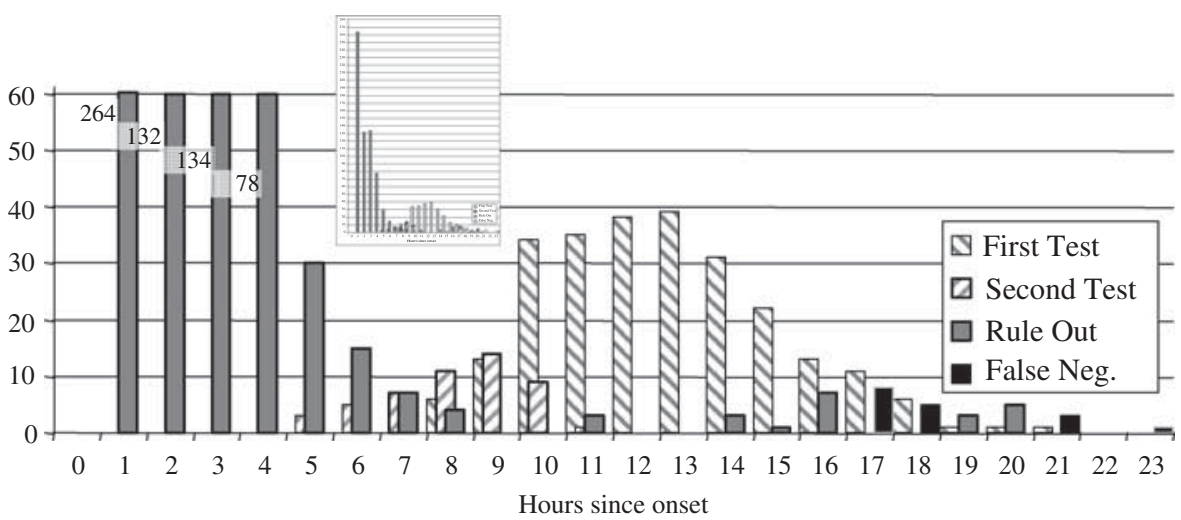

Figure 4 Delay in diagnosis of AMI using the first, second and 120 min rule-out protocols. (histogram cropped to emphasise lower counts - thumbnail to show scale)

level from the healthy profile in Figure 1 and as such, the probability of the patients having a troponin level higher than the cut-off value is $1 \%$, this being the prerequisite for this distribution. It is not known from the literature how the troponin level of a healthy person varies with the period of the rule-out interval of between 90 and $180 \mathrm{~min}$. If the patient is healthy, there is little reason to believe that it would change at all though there are random errors within the PoC device (Purchasing and Supply Agency, 2006) that may give some anomalous readings.

The virtual experiment involving 1000 patients was repeated 100 times for each of the three rule-out protocols. Table 2 gives the percentages that would have been accurately diagnosed by the first test at presentation. Failing diagnosis at presentation, the percentage that would be diagnosed at the second test at the rule-out interval is given. The third column shows the percentage that would be diagnosed by the comparison of the first test and the second, given that neither individually had diagnosed AMI. The fourth column shows the percentage that is misdiagnosed by these protocols.

The most critical value is the false negative diagnosis for each of protocols. The $90 \mathrm{~min}$ rule-out has a sensitivity of $94.9 \%$ that may be compared with $\mathrm{Ng}$ et al (2001) and McCord et al (2001) who found sensitivity of $100 \%$ and $96.9 \%$, respectively, for their $90 \mathrm{~min}$ rule-out. The $180 \mathrm{~min}$ rule-out has $99 \%$ sensitivity. Some $70 \%$ of those presenting are dependent on the second test for diagnosis. This of course requires a delay of a critical $3 \mathrm{~h}$ before the second test result becomes known. Although it is critical that delay is kept to a minimum, the increased accuracy of the test may be important, though of diminishing return value. That is, that the increase in sensitivity may be worth waiting $120 \mathrm{~min}$ for but not $180 \mathrm{~min}$.

It is interesting to review the profile of the patients that would be diagnosed at each stage in Figure 4. Those diagnosed by the first or second test are typically in their $10-15 \mathrm{~h}$ since onset of chest pain which is slightly earlier for those diagnosed by the second test only. Those for whom the diagnosis is a false negative are on average tested $18 \mathrm{~h}$ after onset and typically only have mild AMI. For these patients, the delay of further diagnosis may not be a life-threatening situation. It can be seen that the rule-out comparisons are a most powerful diagnostic test especially where the first two tests alone provided insufficient evidence of AMI. The profile of the patients that were diagnosed by the rule-out were on average tested $2.7 \mathrm{~h}$ since chest pain onset at presentation, much earlier than is generally recommended for a first test. Given the urgency for early diagnosis and treatment with thrombolytic drugs, the rule-out protocols would seem to be a valuable course of action. There is considerable difference in comparison with non-rule-out protocols. Hamilton et al (2008) records a sensitivity of $78.9 \%$ with troponin and Goodacre et al (2005) records a sensitivity with troponin of $83.3 \%$ at a $0.1 \mathrm{ng} / \mathrm{ml}$ cut-off. Both these diagnostic tests would have conducted over $6 \mathrm{~h}$ after onset. 
The evidence from the model suggests that rule-out protocol is a useful tool to have in the toolbox. It is not the only tool however, and it of course has to be used in conjunction with ECG, TIMI scores and with the wisdom and experience accrued by healthcare professionals working many years in cardiac care. It must be pointed out that, however valuable the rapid rule-out protocol is, cardiac markers will not identify disease where there is insignificant myocardial necrosis, such as may be found with unstable angina. Similarly, myoglobin is not specific to cardiac necrosis and may not indicate AMI if it is the only cardiac marker present. Studies by the International Liaison Committee on Resuscitation (2005) suggest that rapid ruleout protocols as suggested above should not be recommend before $6 \mathrm{~h}$ since onset. This would seem to be missing the point of rapid rule-out and to negate its key strength.

\section{Conclusion}

The aim of this paper was to demonstrate that while there may be some apprehension in the use of synthetic data, there is significant value in the practice. Although this exemplar is little more than a proof of concept, it can clearly be seen that the quality of data can be improved with time and experience until it will eventually pass the 'Turing Test' of medical data. Given sufficient true information, methods of principal components or Bayesian belief networks can be used to reproduce probabilistic interdependencies thus allowing diagnostic inference to be learned from the synthetic data.

In a hospital environment the data generated in this analysis would have taken years to accumulate and multiple repetitions would just not have been possible. Using this data to research the use of cardiac marker rule-out protocols permits the exploration of these protocols without endangering lives. It allows one to examine which parameters the model is most sensitive to highlight the information that needs to be gathered and to design better trials to test hypothesis. It also permits the open sharing of data between co-workers so that the experiment will be reproducible and open to scrutiny.

There will always be dangers involved in the use of synthetic data. Where it is seeded from sample live data there is the risk of bias. If the original sample were biased, maybe as a result of being taken from a small sample, the generated data will also reflect that bias. Although this is true, the same problem could as easily arise if real data were used in a pilot study. However, there is here an advantage of synthetic data. Given that such bias will in all probability arise inadvertently anyway, the synthetic data has the ability to test the sensitivity to such a bias by actively loading the data. As mentioned above, the data is not intended to change clinical practice, but to provide a tool to explore issues in clinical practice and design better experiments to verify new procedures. In the words of Alfred North Whitehead, 'Seek simplicity, and distrust it'.

Acknowledgements - The authors acknowledge support of this work through the UK MATCH programme Engineering \& Physical Science Research Council (EPSRC) Grant GR/S29874/01, although the views expressed in this work are entirely their own

\section{References}

Adams P (2008). Definition of acute myocardial infarction Newcastle Hospitals NHS trust, www.c2c.nhs.uk/media/\% 7BC8D D3294-4961-4004-A86F-4F7062438F6A\%7D.ppt, accessed 29 September 2008.

Apple FS et al (2005). Validation of the 99th percentile cutoff independent of assay imprecision $(\mathrm{CV})$ for cardiac troponin monitoring for ruling out myocardial infarction. Clin Chem 51: 2198-2200.

Apple FS et al (2003). Plasma 99th percentile reference limits for cardiac troponin and creatine kinase MB mass for use with European society of cardiology/American college of cardiology consensus recommendations. Clin Chem 49(8): 1331-1336.

Benger JR, Karlsten R and Eriksson B (2002). Prehospital thrombolysis: Lessons from Sweden and their application to the United Kingdom. Emerg Med J 19: 578-583.

Body R (2008). Emergent diagnosis of acute coronary syndromes: Today's challenges and tomorrow's possibilities. Resuscitation 78: $13-20$.

Brendan $\mathrm{M}$ et al (2002). Triage of patients with chest pain in the emergency department: A comparative study of physicians' decisions. Am J Med 112: 95-103.

Carley S et al (2002). What's the point of ST elevation? Emerg Med J 19: 126-128.

Collinson PO et al (2006). Comparison of biomarker strategies for rapid rule-out of myocardial infarction in the emergency department using ACC/ESC diagnostic criteria. Ann Clin Biochem 43: 273-280.

DeVita MA et al (2005). Improving medical emergency team (MET) performance using a novel curriculum and a computerized human patient simulator. Qual Saf Health Care 14: 326-331

Dunn F, Hughes D, Rocke LGR and McNicholl BP (2006). Are chest pain observation units essential for rapid and effective emergency care in the UK? Emerg Med $J$ 23: 487-488.

Dutta P et al (2005). SimCare: A model for studying physician decision making activity. Advances in patient safety: from research to implementation: Programs, Tools, and Products No. 4. Rockville (MD) agency for healthcare research and quality. AHRQ Publication 4(5): 21-24.

Fox KF (2005). Investigation and management of chest pain. Heart 91: $105-110$.

Goodacre S et al (2007). The ESCAPE Multi-Centre evaluation of the role of chest pain units in the NHS. Available at http:// www.sdo.nihr.ac.uk, accessed 2007.

Goodacre S et al (2005). Which diagnostic tests are the most useful in chest pain unit protocol. BMC Emerg Med 5(6): 1-7.

Goodacre $\mathrm{S}$ et al (2004). Randomized controlled trial and economic evaluation of a chest pain observation unit compared with routine care. BMJ 328: 254-257.

Hamilton AJ et al (2008). Risk stratification of chest pain patients in the emergency department by a nurse utilizing a point of care protocol. Eur J Emerg Med 15: 9-15.

Heath SM et al (2003). Nurse intiated thrombolysis in the accident and emergency department. Emerg Med $J$ 20: 418-420. 
Information Governance Statement of Compliance V6.0. NHS Connecting for Health. IGSOC Documents, available at http:// www.connectingforhealth.nhs.uk/systemsandservices/infogov/ igsoc/links, accessed 31 October 2008.

NHS National Workforce Projects, available at http://www. healthcareworkforce.nhs.uk/resource_library/latest_re sources/ workforce_planning_development_menus.html, accessed 31 October 2008.

UK Clinical Research Collaboration (UKCRC) Response to: The Ministry of Justice Consultation on the Use and Sharing of Personal Information in the Public and Private Sector. Office For Strategic Coordination of Health Research. Chairman's First progress Report, November 2008, HMG, available at http:// www.nihr.ac.uk/files/pdfs/oschr_progress_report_18.11.08.pdf, accessed November 2008.

Turning Test, Wikipedia, available at http://en.wikipedia.org/wiki/ Turing_test, accessed 1 February 2009.

International Liaison Committee on Resuscitation (2005). Part 5: Acute coronary syndromes. Resuscitation 67: 249-269.

Lattimer V (2004). Reviewing emergency care systems I: Insights from system dynamics modelling. Emerg Med J 21: 685-691.

McCabe RM (2008). Using Data Mining to Predict Errors in Chronic Disease Care, in Advances in Patient Safety: New
Directions and Alternative Approaches. Agency for Healthcare Research and Quality.

McCord $\mathbf{J}$ et al (2001). Ninety-minute exclusion of acute myocardial infarction by use of quantitative point of care testing of myoglobin and Troponin I. Circulation 104: 1483-1488.

National Institute for Clinical Excellence (NICE) (2002). Guidance on the Use of Drugs for Early Thrombolysis in the Treatment of Acute Myocardial Infarction. London.

$\mathrm{Ng}$ MS et al (2001). Ninety-minute accelerated critical pathway for chest pain evaluation. Am J Cardiol 88: 611-617.

Purchasing and Supply Agency (2006). Three point of care devices for troponin measurement. Evaluation. Report 06020.

Rajappan K et al (2005). Usage of troponin in the real world: A lesson for the introduction of biochemical assays. $Q J \mathrm{Med} \mathbf{9 8}$ : $337-342$.

Turab A, Scrafton J and Andrews R (2006). Near patient testing for cardiac troponin I to reduce hospital stay in patients presenting with chest pain. Br J cardiol (Acute Interv Cardiol) 13: $19-21$.

Received 31 October 2008; accepted 8 April 2009 